\title{
NUMERICAL RANGES OF POWERS OF HERMITIAN ELEMENTS
}

\author{
by M. J. CRABB and C. M. McGREGOR
}

(Received 18 October, 1984)

Introduction An element $k$ of a unital Banach algebra $A$ is said to be Hermitian if its numerical range

$$
V(k)=\left\{\psi(k): \psi \in A^{\prime},\|\psi\|=\psi(1)=1\right\}
$$

is contained in $\mathbb{R}$; equivalently, $\left\|e^{i t k}\right\|=1(t \in \mathbb{R})$-see Bonsall and Duncan [3] and [4]. Here we find the largest possible extent of $V\left(k^{n}\right), n \in \mathbb{N}$, given $V(k) \subseteq[-1,1]$, and so $\|k\| \leqslant 1$ : previous knowledge is in Bollobás [2] and Crabb, Duncan and McGregor [7]. The largest possible sets all occur in a single example. Surprisingly, they all have straight line segments in their boundaries. The example is in [2] and [7], but here we give A. Browder's construction from [5], partly published in [6]. We are grateful to him for a copy of [5], and for discussions which led to the present work. We are also grateful to $\mathrm{J}$. Duncan for useful discussions.

Let $X$ be the Banach space of entire functions $f$ such that

$$
\|f\|=\sup \left\{|f(\sigma+i t)| e^{-|t|}: \sigma, t \text { real }\right\}<\infty .
$$

For $f \in X$, we have $\|f\|=\sup \{|f(\sigma)|: \sigma$ real $\}$-see [6] for proofs here. Define $h$ by $h(f)=i f^{\prime}$. Then $h \in B(X), h$ is Hermitian and $\|h\|=1$. Denote $\{x \in X:\|x\| \leqslant 1\}$ by $X_{1}$.

Lemma 1. If $f \in X_{1}$ and $f(0)=1$, then for $T \in B(X)$ we have $(T f)(0) \in V(T)$.

Proof. Define the functional $\phi$ on the Banach algebra $B(X)$ by $\phi(T)=(T f)(0)$. Then $|\phi(T)| \leqslant\|T f\| \leqslant\|T\|$, so $\|\phi\| \leqslant 1$. Also, $\phi(I)=1$. Hence $\phi(T) \in V(T)$. q.e.d.

Let $k$ be a Hermitian element of a unital Banach algebra $A$ with $\|k\| \leqslant 1$. Let $\psi \in A^{\prime}$ with $\|\psi\|=\psi(1)=1$, and let $f(z)=\psi\left(e^{-i z k}\right)$. Then $f \in X_{1}$ and $f(0)=1$. For $\phi$ as in the above proof, we have $\phi\left(h^{n}\right)=i^{n} f^{(n)}(0)=\psi\left(k^{n}\right)(n=0,1,2, \ldots)$. Hence $V(p(k)) \subseteq$ $V(p(h))$ for any polynomial $p$. The same argument with the restrictions $\psi(1)=\phi(I)=1$ removed shows that $\|p(k)\| \leqslant\|p(h)\|$.

The next two theorems contain the main results of the paper. They are proved in the sequel.

THEOREM 2 (even power case). Let $\zeta(\theta)$ be the $2 n$-th derivative at 0 , with respect to $x$, of $e^{-i \theta}\left(\cos \rho+i \theta \rho^{-1} \sin \rho\right)$, where $\rho^{2}=x^{2}+\theta^{2}$. Then the boundary of $V\left(h^{2 n}\right)$ consists of the curves $\zeta(\theta)$ and $\overline{\zeta(\theta)}, 0 \leqslant \theta \leqslant \pi$, and the line segment $[\zeta(\pi), \overline{\zeta(\pi)}]$.

THEOREM 3 (odd power case). Let $\zeta(\theta)$ be the $(2 n+1)$-th derivative at 0 , with respect to $z$, of $i^{2 n+1} e^{-i A}\left(\cos Q+i\left(A+A^{-1} \alpha z\right) Q^{-1} \sin Q\right)$, where $A=\sqrt{\theta^{2}+\alpha^{2}}$ and $Q^{2}=(z+\alpha)^{2}+\theta^{2}, \alpha$ being the first positive zero of $\left(d^{2 n} / d z^{2 n}\right)(\sin \rho / \rho)$ with $\rho^{2}=z^{2}+\theta^{2}$. Let $\theta_{0}$ be the first positive $\theta$ for which $A=\pi$. Then the boundary of $V\left(h^{2 n+1}\right)$ consists of the curves $\pm \zeta(\theta)$ and $\pm \overline{\zeta(\theta)}, 0 \leqslant \theta \leqslant \theta_{0}$, and the line segments $\left[ \pm \zeta\left(\theta_{0}\right), \mp \overline{\zeta\left(\theta_{0}\right)}\right]$.

Glasgow Math. J. 28 (1986) 37-45. 


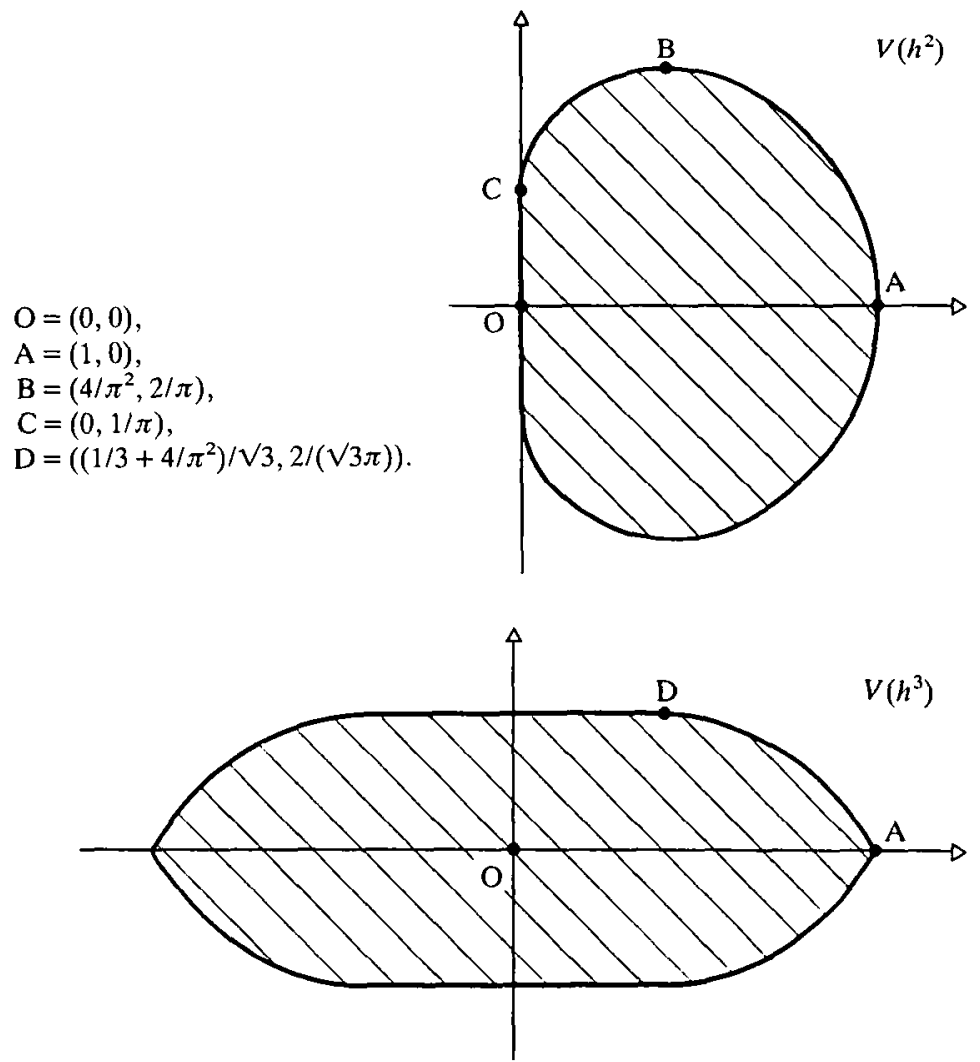

Figure 1

Figure 1 illustrates Theorems 2 and 3 with $n=1$.

LEMMA 4. For any polynomial $p,\|p(h)\|=\sup \left\{|(p(h) f)(0)|: f \in X_{1}\right\}$.

Proof. Write $m$ for the sup. By considering $f(s+u)$ for $f \in X_{1}$, we obtain $|(p(h) f)(u)| \leqslant m$. So $\|p(h)\| \leqslant m$, and the reverse inequality is clear. q.e.d.

For any $a \in B(X)$, we have $V(a) \subseteq\{\zeta:|\zeta| \leqslant\|a\|\}$. Hence if $\zeta \in V(a)$ and $|\zeta|=\|a\|$, then $\zeta \in \partial V(a)$. Also, $V(a+\gamma)=V(a)+\gamma(\gamma \in \mathbb{C})$.

The even power case. Define, for $\theta, z \in \mathbb{C}, f_{\theta}(z)=\cos \rho=\sum_{n=0}^{\infty}(-1)^{n}\left(z^{2}+\theta^{2}\right)^{n} /(2 n)$ !, where $\rho=\sqrt{z^{2}+\theta^{2}}$. We can take either square root and get the same value for $f_{\theta}$. Observe that $f_{\theta}^{\prime}(z)=-z g_{\theta}(z)$, where $g_{\theta}(z)=\sin \rho / \rho$. For $\theta \geqslant 0, f_{\theta}$ and $g_{\theta}$ are even functions in $X_{1}$. To see this, apply Lemma 3.2 of [7] to the function $\phi(w, z)=f_{i w}(i z)$. This gives $f_{\theta} \in X_{1}$ for $\theta \in \mathbb{R}$. Then $\sin \rho / \rho=\int_{0}^{1} \cos (u \rho) d u \in X_{1}$.

Let $0 \leqslant \theta<\pi$, and put $e=f_{\theta}$. Consider, for $f$ in $X, \int_{\Gamma_{j}} F(z) d z$, where 


$$
F(z)=p(z) f(z) /\left(z^{2 n} e^{\prime}(z)\right)=-p(z) f(z) /\left(z^{2 n+1} g_{\theta}(z)\right) \text {. }
$$

Here $\Gamma_{j}$ is the square with corners $\left(j+\frac{1}{2}\right) \pi( \pm 1, \pm i)$, and

$$
p(z)=\sum_{k=0}^{2 n-2} \frac{g^{(k)}(0)}{k !} z^{k} \text {. }
$$

On $\Gamma_{j},|\sin z|>\frac{1}{3} e^{|t|}$, where $z=\sigma+i t$. Hence $|\sin \rho|=|\sin (z+w)|$, where $|w| \rightarrow 0$ as $|z| \rightarrow \infty,>|\sin z \cos w|-|\cos z \sin w|>\frac{1}{4} e^{|t|}$ for all large enough $|z|$, since $|\cos z| \leqslant e^{|t|}$. Since $|z / \rho| \rightarrow 1$ as $|z| \rightarrow \infty$, we get $\left|e^{\prime}(z)\right|>\frac{1}{5} e^{|t|}$ for all large $|z|$. Hence $\int_{\Gamma_{i}} F d z \rightarrow 0$ as $j \rightarrow \infty$, and the sum of the residues of $F$ is 0 . The function $F$ is meromorphic with poles at 0 and at $\left\{\alpha_{k}\right\} \subset \mathbb{R}$, the zeros of $g_{\theta}$. Also, $g_{\theta}(z)=p(z)+z^{2 n} q(z)$, where $q$ is entire. So

$$
\frac{p(z)}{g_{\theta}(z)}=\left(1+\frac{q(z)}{p(z)} z^{2 n}\right)^{-1}=1-\frac{q(0)}{p(0)} z^{2 n}+\ldots=1-\frac{g^{(2 n)}(0)}{g(0)} \frac{z^{2 n}}{(2 n) !}+\ldots
$$

Hence the residue of $F$ at 0 is $-(1 /(2 n) !)\left(f^{(2 n)}(0)-\tau f(0)\right)$, where $\tau=g_{\theta}^{(2 n)}(0) / g_{\theta}(0)$. Therefore, where we are defining $\phi \in X^{\prime}$,

$$
\phi(f)=\left((i h)^{2 n}-\tau\right) f(0)=f^{(2 n)}(0)-\tau f(0)=(2 n) ! \sum_{k} \frac{p\left(\alpha_{k}\right) f\left(\alpha_{k}\right)}{\alpha_{k}^{2 n} e^{\prime \prime}\left(\alpha_{k}\right)} .
$$

At $z=\alpha_{k}, \sin \rho=0$, so $e=\cos \rho= \pm 1$, and $e, e^{\prime \prime}$ have opposite signs: note that $e(\mathbb{R})=[-1,1]$. Hence for all $k, e\left(\alpha_{k}\right) / e^{\prime \prime}\left(\alpha_{k}\right)<0$. Thus $|\phi(e)|=\max \left\{|\phi(f)|: f \in X_{1}\right\}$, attaining the estimate $(2 n) ! \sum_{k} \alpha_{k}^{-2 n}\left|p\left(\alpha_{k}\right)\right| /\left|e^{\prime \prime}\left(\alpha_{k}\right)\right|$, if the $p\left(\alpha_{k}\right)$ have constant sign. For $n=1$ this follows since $p$ is constant. For $n>1$, it is proved later.

Thus by Lemma $4,|\phi(e)|=\left\|(-1)^{n} h^{2 n}-\tau\right\|=\left\|h^{2 n}-\tau^{\prime}\right\|$, where $\tau^{\prime}=(-1)^{n} \tau$. Define $k_{\theta}(z)=k(z)=e^{-i \theta}(\cos \rho+i \theta \sin \rho / \rho)=e^{-i \theta}\left(f_{\theta}(z)+i \theta g_{\theta}(z)\right)$. Then $k \in X$, and $|k| \leqslant 1$ on $\mathbb{R}$, so $k \in X_{1}$. Since $k(0)=1, \zeta=\xi(\theta)=(-1)^{n} k^{(2 n)}(0) \in V\left(h^{2 n}\right)$. By the definition of $\phi$ and $\tau, \phi\left(g_{\theta}\right)=0$. Thus $(-1)^{\prime \prime}\left(\zeta-\tau^{\prime}\right)=k^{(2 n)}(0)-\tau=\phi(k)=e^{-i \theta} \phi\left(f_{\theta}\right)$, and $\left|\zeta-\tau^{\prime}\right|=$ $\left|\phi\left(f_{\theta}\right)\right|=\left\|h^{2 n}-\tau^{\prime}\right\|$. Since $\zeta-\tau^{\prime} \in V\left(h^{2 n}-\tau^{\prime}\right)$, we get $\zeta \in \partial V\left(h^{2 n}\right)$. Also, $V\left(h^{2 n}-\tau^{\prime}\right) \subseteq$ $\left\{z:|z| \leqslant\left|\zeta-\tau^{\prime}\right|\right\}$. Hence $V\left(h^{2 n}\right)$ is contained in a circle with centre at $\tau^{\prime}$ and through $\zeta$.

As $\theta \rightarrow \pi, g_{\theta}(0)=\sin \theta / \theta \rightarrow 0$. We prove below that $g_{\theta}^{(2 n)}(0) \neq 0$ for $0 \leqslant \theta \leqslant \pi$. These are continuous in $\theta$, and so $\left|\tau^{\prime}\right|=|\tau| \rightarrow \infty$ as $\theta \rightarrow \pi$. Also, $\zeta(\theta) \rightarrow \zeta(\pi)=\zeta_{0}$ say, which is also in $V\left(h^{2 n}\right)$ and $\operatorname{Im}\left(\zeta_{0}\right)=-\pi(-1)^{n} g_{\pi}^{(2 n)}(0) \neq 0$ (below).

The function $\overline{k(\bar{z})}$ gives $\bar{\zeta}$ and $\bar{\zeta}_{0}$ in $\partial V\left(h^{2 n}\right)$. Hence the line segment $\left[\zeta_{0}, \bar{\zeta}_{0}\right\rfloor \subseteq V$. The discs with centre $\tau^{\prime}$ and through $\zeta$ tend, as $\theta \rightarrow \pi$, to a half-plane with edge through $\zeta_{0}$ and $\bar{\zeta}_{0}$, which also contains $V$. Thus $\left[\zeta_{0}, \bar{\zeta}_{0}\right] \subseteq \partial V\left(h^{2 n}\right)$. Since $f_{\theta}(z)$ and $g_{\theta}(z)$ are continuous in $\theta$ and $z, \zeta(\theta)$ for $0 \leqslant \theta \leqslant \pi$ is a continuous curve $C$ in $\partial V$. For $\theta=0$, we have $k(z)=\cos z$, and $\zeta=1$. So $C$ runs from 1 to $\zeta_{0}$. The curve $\bar{C}$ is continuous from 1 to $\bar{\zeta}_{0}$, so with $C$ and $\left[\xi_{0}, \bar{\zeta}_{0}\right]$, we have a closed curve which must be all of $\partial V\left(h^{2 n}\right)$, since $V\left(h^{2 n}\right)$ is a convex set.

From above, $\zeta-\tau^{\prime}=(-1)^{n} e^{-i \theta} \phi\left(f_{\theta}\right)$, and $\phi\left(f_{\theta}\right)$ is real. For $\theta=\frac{\pi}{2}$ we get $\zeta=\tau^{\prime}+i \eta$ with $\eta$ real. $V\left(h^{2 n}\right)$ is contained in the circle with centre $\tau^{\prime}$ and through $\zeta$. Hence $\max \left\{|\operatorname{Im} z|: z \in V\left(h^{2 n}\right)\right\}=|\eta|$, and occurs at $\zeta$. Also, if $\sigma$ is real and $\neq \tau^{\prime}$, then since 
$\zeta-\sigma \in V\left(h^{2 n}-\sigma\right)$, we get $\quad\left\|h^{2 n}-\sigma\right\| \geqslant|\zeta-\sigma|>\left|\zeta-\tau^{\prime}\right|=\left\|h^{2 n}-\tau^{\prime}\right\|$. Thus $\min \left\{\left\|h^{2 n}-\sigma\right\|: \sigma \in \mathbb{R}\right\}$ occurs at $\sigma=\tau^{\prime}$.

We can prove that $\sup \left\{\operatorname{Re} e^{-i \theta_{z}} z: z \in V\left(h^{2 n}\right)\right\}=(-1)^{n} f_{\theta}^{(2 n)}(0)(0 \leqslant \theta \leqslant \pi)$. For $V\left(h^{2}\right)$, this is $\sin \theta / \theta$. This was found first by $\mathrm{J}$. Duncan, who also pointed out that $\frac{1}{2} V\left(h^{2}\right)=W(T)$, the numerical range of the Volterra operator on $L^{2}(0,1)$-see Halmos [8, p. 109].

The following completes the proof of Theorem 2 . $\geqslant 2$.

Lemma 5. For $0 \leqslant \theta<\pi, g_{\theta}$ has the following property, for degrees of polynomial

A partial sum (polynomial) of the power series at 0 is, on $\mathbb{R}$, either always $\geqslant$ the function, or always $\leqslant$ the function.

Hence, at the zeros of $g_{\theta}$ the polynomial has constant sign.

Proof. The functions $\cos x$ and $\sin x / x$ have property (2) for all degrees (e.g. Hardy [9, ExxXLVI, 5]). This gives (2) for $\theta=0$, so assume now that $\theta>0$.

We have $g^{\prime}(x)=-x k(x)$, where $k(x)=(\sin \rho-\rho \cos \rho) / \rho^{3}=\sqrt{\pi / 2} \rho^{-3 / 2} J_{3 / 2}(\rho)$, and $J_{n}$ is the usual Bessel function. From Luke [10, p. 299, Eqn. (26)], for $\operatorname{Re} \mu>-1$, $\operatorname{Re} v>-1$,

$$
\int_{0}^{\pi / 2} J_{\mu}(\theta \sin t) J_{v}(x \cos t) \sin ^{\mu+1} t \cos ^{v+1} t d t=\theta^{\mu} x^{v} J_{\mu+v+1}(\rho) / \rho^{\mu+v+1} .
$$

If we put $\mu=1, v=-\frac{1}{2}$, we get $k(x)=\theta^{-1} \int_{0}^{\pi / 2} \cos (x \cos t) J_{1}(\theta \sin t) \sin ^{2} t d t$. It is enough to prove (2) for $k$ and its polynomials of degree $\geqslant 0$ : we multiply by $x$ and integrate to establish (2) for $g$. As $k$ is an integral of functions $x \rightarrow \alpha \cos (\beta x)$ with $\alpha>0$, each of which satisfies (2) in the same direction for any degree, it follows that $k$ satisfies (2). q.e.d.

The above also gives $g_{\theta}^{(2 n)}(0)=-(2 n-1) k^{(2 n-2)}(0)$, and

$$
(-1)^{k} k^{(2 k)}(0)=\theta^{-1} \int_{0}^{\pi / 2} \cos ^{2 k} t \sin ^{2} t_{1}(\theta \sin t) d t>0,
$$

since $J_{1}>0$ on $\left.] 0, \pi\right]$. Hence $g_{\theta}^{(2 n)}(0) \neq 0$, for $n \in \mathbb{N}$ and $0<\theta \leqslant \pi$.

Remarks. To see that $\sup \operatorname{Re} e^{-i \theta} V\left(h^{2 n}\right)=(-1)^{n} f_{\theta}^{(2 n)}(0)$, note that with the above notation, in the disc centred at $\tau^{\prime}$ which contains $V\left(h^{2 n}\right)$ and has $\zeta$ in $V\left(h^{2 n}\right)$ on its boundary, we have that the segment $\left[\tau^{\prime}, \zeta\right]$ makes an angle $\theta$ with the real axis. Hence the tangent to the circle at $\zeta$ is a support line of $V\left(h^{2 n}\right)$.

We can prove that

$$
f_{\theta}(x)=\cos \rho=\cos x-\int_{0}^{\pi / 2} \theta \cos (x \cos t) J_{1}(\theta \sin t) d t .
$$

This gives $(-1)^{n} f_{\theta}^{(2 n)}(0)=1-\theta \int_{0}^{\pi / 2} \cos ^{2 n} t J_{1}(\theta \sin t) d t$. Since $J_{1}(\theta \sin t)>0,(-1)^{n} f^{(2 n)}(0)$ increases monotonically to 1 as $n \rightarrow \infty$. So the $V\left(h^{2 n}\right)$ expand up to the unit disc.

For $V\left(h^{2}\right)$, the line segment in the boundary is $[-i / \pi, i / \pi]$. The point $4 / \pi^{2}+2 i / \pi$ gives $\max \left\{|\operatorname{Im} z|: z \in V\left(h^{2}\right)\right\}$.

Functions $e(z)=\cos \rho$ for $\theta \geqslant \pi$ are also "extremal functions". For instance, if for 
$\pi<\theta<2 \pi$ we integrate $\left(z^{2}+\theta^{2}-\pi^{2}\right) f(z) /\left(z^{4} e^{\prime}(z)\right)$ as above, we find the norm in $X$ of $h^{4}+\xi h^{2}+\eta$ for certain $\xi, \eta$.

The odd power case. For $f$ in $X$, consider

$$
\int_{\Delta_{i}} \frac{f(z) p(z)}{z^{2 n+2} e^{\prime}(z)} d z
$$

with $e(z)=\cos \sqrt{(z+\alpha)^{2}+\theta^{2}}=f_{\theta}(z+\alpha)$ for certain $\alpha>0,0 \leqslant \theta<\pi$, and $p$ the $2 n$ degree polynomial which starts the power series of $e^{\prime}$. Take $\Delta_{j}=\Gamma_{j}-\alpha, \Gamma_{j}$ as before, and let $j \rightarrow \infty$. This gives

$$
\phi(f)=f^{(2 n+1)}(0)-\tau f(0)=-(2 n+1) ! \sum_{k} \frac{p\left(\alpha_{k}\right) f\left(\alpha_{k}\right)}{\alpha_{k}^{2 n+2} e^{n}\left(\alpha_{k}\right)}
$$

where $\left\{\alpha_{k}\right\}$ are the zeros of $e^{\prime}, \tau=e^{(2 n+2)}(0) / e^{\prime}(0)$, and we are defining $\phi \in X^{\prime}$. Then $|\phi(e)|=\max \left\{|\phi(f)|: f \in X_{1}\right\}$ if $p\left(\alpha_{k}\right)=0$ when $\left|e\left(\alpha_{k}\right)\right| \neq 1$, i.e. for $\alpha_{k}=-\alpha$, and

$$
p\left(\alpha_{k}\right) \text { has the same sign at all } \alpha_{k} \neq-\alpha \text {. }
$$

For then $|\phi(e)|$ attains the estimate

$$
(2 n+1) ! \sum \alpha_{k}^{-2 n-2}\left|p\left(\alpha_{k}\right) / e^{\prime \prime}\left(\alpha_{k}\right)\right| .
$$

Since $-e^{\prime}(x)=(x+\alpha) g_{\theta}(x+\alpha)=(x+\alpha) \sum_{k=0}^{\infty} a_{k} x^{k}$ (say), we have

$$
-p(x)=(x+\alpha)\left(a_{0}+\ldots+a_{2 n-1} x^{2 n-1}\right)+\alpha a_{2 n} x^{2 n} .
$$

We require $p(-\alpha)=0$, i.e. $g_{\theta}^{(2 n)}(\alpha)=a_{2 n}=0$. Then $-p(x)=(x+\alpha)\left(a_{0}+\ldots+\right.$ $\left.a_{2 n-1} x^{2 n-1}\right)$. This is to have constant sign at the zeros of $g_{\theta}(x+\alpha)$. Put $t=x+\alpha$. We require $t r_{2 n-1}(t)$ to have constant sign at the zeros of $g_{\theta}(t)$, where

$$
r_{2 n-1}(t)=\sum_{k=0}^{2 n-1} \frac{g^{(k)}(\alpha)}{k !}(t-\alpha)^{k}
$$

Let $\beta=\sqrt{\pi^{2}-\theta^{2}}$, the first positive zero of $g_{\theta}$. We prove the following, for $n \geqslant 2$ and certain $\theta$. There exists $\alpha, 0<\alpha<\beta$, such that $g_{\theta}^{(2 n)}(\alpha)=0$ and $\left(r_{2 n-1}-g_{\theta}\right)(t)$ has one sign for $t<\alpha$, the opposite sign for $t>\alpha$ : we say that $r_{2 n-1}$ crosses $g_{\theta}$ at $\alpha$. Therefore $t\left(r_{2 n-1}-g_{\theta}\right)(t)$ has the same sign for $\left.t \in\right]-\infty, 0[U] \alpha, \infty[$, which set contains the zeros of $g_{\theta}$. Hence at these zeros, $t_{2 n-1}(t)=t\left(r_{2 n-1}-g_{\theta}\right)(t)$ has constant sign. The case $n=1$ is considered later.

In (3) we put $\mu=0$ and $v=-\frac{1}{2}$. This gives, after substitution for $\cos t$,

$$
g_{\theta}(x)=\sin \rho / \rho=\int_{0}^{1} \cos (x t) J_{0}\left(\theta \sqrt{1-t^{2}}\right) d t .
$$

Fix $n \in \mathbb{N}$. Let $G_{\theta}(x)=(-1)^{n} g_{\theta}^{(2 n)}(x)$. Hence $G_{\theta}(x)=\int_{0}^{1} \cos (x t) w_{n}(t) d t$, where $w_{n}(t)=t^{2 n} J_{0}\left(\theta \sqrt{1-t^{2}}\right)$. Our method is as follows. We find $0<\alpha<\pi$ such that $g_{\theta}^{(2 n+2)}(\alpha)=G_{\theta}^{\prime \prime}(\alpha)=0$. Define $T(x)=G(\alpha)+(x-\alpha) G^{\prime}(\alpha)$. We prove that $T$ crosses $G$ at $\alpha$. Integrating this $2 n$ times, we find that $r_{2 n+1}$ crosses $g_{\theta}$ at $\alpha$. 
Since $J_{0}$ decreases on $[0, \pi], w_{0}(t)=J_{0}\left(\theta \sqrt{1-t^{2}}\right)$ increases on $[0,1]$. Let $z_{1}$ be the first zero of $J_{0}$, so $z_{1} \bumpeq 2 \cdot 4$. When $\theta>z_{1}$, let $a=\sqrt{1-\left(z_{1} / \theta\right)^{2}}$. Then $w_{n}(t)<0(0 \leqslant t<a)$, and

$$
\text { on }] a, 1], w_{n} \text { is positive and increasing. }
$$

When $\theta \leqslant z_{1}$, take $a=0$, so (6) is still valid.

Lemma 6. Let $k:[0,1] \rightarrow \mathbb{R}^{+}$be continuous, $k \neq 0$. Then, for $m, j \in \mathbb{Z}, 0 \leqslant j<m$,

$$
\int_{0}^{1} k(t) t^{m} w_{0}(t) d t>a^{m-j} \int_{0}^{1} k(t) t^{j} w_{0}(t) d t .
$$

Proof. We have $k(t) w_{0}(t)\left(t^{m}-a^{m-j} t^{j}\right) \geqslant 0$, with strict inequality for some $t$. q.e.d.

For $\theta=0$, we shall see in (8), (12) that $G^{\prime \prime}$ has a zero $\alpha$ with $0<\alpha<\beta=\pi$.

THEOREM 7 (Laguerre [1] p. 23). Let $f$ be an entire function, real on $\mathbb{R}$, with $e^{-|z|} f(z)$ bounded and all the zeros of $f$ real and simple. Then the zeros of $f^{\prime}$ are real and simple, and interlace the zeros of $f$.

Hence this also applies to $f^{(n)}$ in place of $f$. Note that $g_{\theta}$ satisfies the conditions of Theorem 7, and hence we can apply it to $G^{\prime}$. Since $G^{\prime}(0)=0$, we get a unique zero $\alpha(\theta)$ of $G^{\prime \prime}$ between 0 and the first positive zero of $G^{\prime}$. By Hurwitz's theorem. $\alpha(\theta)$ is continuous. Define $A=\sqrt{\alpha^{2}+\theta^{2}} ; A(\theta)$ is continuous. If $A<\pi$, then $\alpha<\sqrt{\pi^{2}-\theta^{2}}=\beta$. We shall see in (7) that for $\theta>(\sqrt{3} / 2) \pi$, we have $\alpha>\beta$ and $A>\pi$. We let $\theta$ increase from 0 till the first value $\theta_{0}$ with $A=\pi$. We shall prove that for each $0 \leqslant \theta<\theta_{0}$, the function $T$ crosses $G$ at $\alpha$, and since also $\alpha<\beta$, these values of $\theta, \alpha$ give that $|\phi(e)|$ is the maximum of $|\phi(f)|$ for $f$ in $X_{1}$.

Suppose that $(\sqrt{3} / 2) \pi<\theta<\pi$. If $0 \leqslant x \leqslant \beta$, then $x<\pi / 2$. Since

$$
g_{\theta}(x)=\int_{0}^{1} \cos (x t) w_{0}(t) d t>0,
$$

and $\cos (x t)>0$, here, Lemma 6 gives $G(x)>a^{2 n} g_{\theta}(x) \geqslant 0$. This inequality for $n$ replaced by $n+1$ is $-G^{\prime \prime}(x)>0$. Thus $G^{\prime \prime}$ has no zero for $0 \leqslant x \leqslant \beta$, and so $\alpha>\beta$. Hence

$$
\theta \leqslant \frac{\sqrt{3}}{2} \pi \text { if } \alpha<\beta \text {. }
$$

This argument also shows that for $0 \leqslant \theta \leqslant(\sqrt{3} / 2) \pi$ and $0 \leqslant x \leqslant \pi / 2$, since $g_{\theta}(x) \geqslant 0$ we have

$$
G(x)>0>G^{\prime \prime}(x) \quad\left(0 \leqslant x \leqslant \frac{\pi}{2}\right) .
$$

Henceforth we assume that $\theta \leqslant(\sqrt{3} / 2) \pi$. Therefore $(\sqrt{3} / 2) \theta \leqslant \frac{3}{4} \pi<z_{1}$, and this gives $a<\frac{1}{2}$. For $0<x<\pi, g_{\theta}^{\prime}(x)=(\rho \cos \rho-\sin \rho) x / \rho^{3}<0$ since $0<\rho<\sqrt{7} \pi / 2<$ the first positive root of $\tan \rho=\rho$. Hence by Lemma 6 ,

$$
-G^{\prime}(x)=\int_{0}^{1} \sin (x t) t^{2 n+1} w_{0}(t) d t>a^{2 n} \int_{0}^{1} \sin (x t) t w_{0}(t) d t=-a^{2 n} g_{\theta}^{\prime}(x) \geqslant 0 .
$$


For $n$ replaced by $n+1$ we get $G^{(3)}(x)>0$. Thus we have

$$
G^{\prime}(x)<0<G^{(3)}(x) \quad(0<x<\pi) .
$$

Let $0 \leqslant \theta<\theta_{0}$, so that $0<\alpha<\beta$ with $G^{\prime \prime}(\alpha)=0$. Since $G^{(3)}>0$ on $] 0, \pi$, we have

$$
G^{\prime}(\alpha)=\min \left\{G^{\prime}(x): 0 \leqslant x \leqslant \pi\right\} .
$$

Hence

$$
T(x)>G(x) \quad(0 \leqslant x<\alpha), \quad T(x)<G(x) \quad(\alpha<x \leqslant \pi) .
$$

Now put $y=\pi /(1+a)$. For $0<t<\frac{1}{2}(1+a)$, we have $\cos (y t)>0$. The substitution $s=1+a-t$ gives

Hence

$$
\int_{(1+a) / 2}^{1} \cos (y t) w_{n}(t) d t=-\int_{a}^{(1+a) / 2} \cos (y s) w_{n}(1+a-s) d s .
$$

$$
\int_{a}^{1} \cos (y t) w_{n}(t) d t=\int_{a}^{(1+a) / 2} \cos (y t)\left(w_{n}(t)-w_{n}(1+a-t)\right) d t<0
$$

by (6), since $a \leqslant t \leqslant 1+a-t$ in the last integral. $\int_{0}^{a} \cos (y t) w_{n}(t) d t \leqslant 0$ since here $w_{n}(t) \leqslant 0$. We add these inequalities to get $G(y)<0$. By $(9)$, we deduce that

$$
G(x)<0<G^{\prime \prime}(x) \quad(\pi /(1+a) \leqslant x \leqslant \pi),
$$

since $n$ replaced by $(n+1)$ gives the $G^{\prime \prime}$ inequality. Now by (8) and (12), $\alpha<y$. Since $G(y)<0<G(0)$, we have by $(10),-G^{\prime}(\alpha)>y^{-1} G(0)$. By $(11), T(y)<G(y)<0$. Since $T$ has slope $G^{\prime}(\alpha)$, we deduce that

$$
T(x)<-(1+2 a) G(0) \quad(x \geqslant 2 \pi) .
$$

We claim the following.

For each $\pi<x<2 \pi$, there exists $0<w \leqslant \pi$ such that $G^{\prime}(w)<G^{\prime}(x)$.

To prove this let $c=\pi / x$ and $b=\pi /(\pi+x)$ so $a<\frac{1}{2}<c<1$. We have $-G^{\prime}(x)=$ $\int_{0}^{1} \sin (x t) v(t) d t$, where $v(t)=t w_{n}(t)$. Consider first the case $a \leqslant b$. Since $v$ increases on $[a, 1], \int_{a}^{c} \sin (x t) v(t) d t=c \int_{a / c}^{1} \sin (\pi s) v(c s) d s<\int_{a}^{1} \sin (\pi s) v(s) d s$. For $0<t<a, \frac{1}{2}(x+\pi) t$ $<\pi a /(2 b) \leqslant \pi / 2$, and so $\sin (x t)-\sin (\pi t)=2 \sin \frac{x-\pi}{2} t \cos \frac{x+\pi}{2} t>0$. Since $v$ is negative on $\left[0, a\left[\right.\right.$, we get $\int_{0}^{a}(\sin (x t)-\sin (\pi t)) v(t) d t \leqslant 0$. For $c<t<1$, we have $v(t)>0$ and $\sin (x t)<0$. Hence $-G^{\prime}(x)<\int_{0}^{c} \sin (x t) v(t) d t<\int_{0}^{1} \sin (\pi s) v(s) d s$ (add the above inequalities) $=-G^{\prime}(\pi)$. Thus we can take $w=\pi$.

Now suppose that $a>b$. Let $w=\pi a^{-1}-x$. Then $w>0$ since $a x<\pi$, and $\left.\pi-w\right\rangle$ $\pi+x-\pi b^{-1}=0$. Since $(x+w) a=\pi$ and $x+w<3 \pi$, we deduce that $\sin (x t)-\sin (w t)>$ 0 if $0<t<a$, and $<0$ if $a<t<1$. Hence $(\sin (x t)-\sin (w t)) v(t) \leqslant 0$ for $0<t<1$, and $-G^{\prime}(x)+G^{\prime}(w)<0$. Thus $(14)$ is established.

Now by (10) and (14), $G^{\prime}(x)>G^{\prime}(\alpha)$ for $\pi<x<2 \pi$. Since $T(\pi)<G(\pi)$ by (11), we have $T(x)<G(x)(\pi \leqslant x \leqslant 2 \pi)$. 
Now consider the case $n=1$, i.e.

$$
G(x)=-g_{\theta}^{\prime \prime}(x)=\int_{0}^{1} \cos (x t) w_{1}(t) d t ; \quad w_{1}(t)=t^{2} w_{0}(t)
$$

Suppose that $a>0$, i.e. $\theta>z_{1}$. Since $\left|J_{0}\right| \leqslant 1$, $\left|w_{0}\right| \leqslant 1$. Let $A=\int_{0}^{a}\left|w_{1}\right|$ and $B=\int_{a}^{1} w_{1}$. Then $A<\int_{0}^{a} t^{2} d t=a^{3} / 3<a / 12$ and

$$
B-A=\int_{0}^{1} w_{1}=G(0)=-\cos \theta / \theta^{2}+\sin \theta / \theta^{3}>-4 \cos z_{1} /\left(3 \pi^{2}\right)>1 / 12 .
$$

Hence $B / A>1+a^{-1}$, and $(B+A) /(B-A)<1+2 a$. For all real $x$,

$$
|G(x)| \leqslant \int_{0}^{1}\left|w_{1}\right|=B+A=G(0)(B+A) /(B-A)<(1+2 a) G(0) .
$$

Hence by (13), $T(x)<G(x)(x>2 \pi)$.

Since $G$ is an even function, (9) shows that $G$ increases on $[-\pi, 0]$. Since $T(0)>G(0)$, we have $T(x)>G(x)(-\pi \leqslant x \leqslant 0)$. Since $-\pi G^{\prime}(\alpha)>(1+a) G(0)$, for $x<-\pi$ we have $T(x)>(2+a) G(0)>(1+2 a) G(0)>G(x)$. This completes the proof that $T$ crosses $G$ if $a>0$ and $n=1$. For $n>1$ and $a>0$, the corresponding ratio $B / A$ is larger, $(B+A) /(B-A)$ smaller, and the above still shows that $T$ crosses $G$ at $\alpha$.

Consider the case $a=0$, i.e. $\theta \leqslant z_{1}$. By the above, $T$ crosses $G$ on $[-\pi, 2 \pi]$, and $|T(x)|>(1+2 a) G(0)=G(0)$ for $x \in \mathbb{R} \backslash[-\pi, 2 \pi]$. Since $w_{n} \geqslant 0$ now, for real $x$, $|G(x)| \leqslant \int_{0}^{1} w_{n}=G(0)$. Thus $|T(x)|>|G(x)|(x \in \mathbb{P} \backslash[-\pi, 2 \pi])$, and $T$ crosses $G$ (on $\mathbb{R}$ ).

Having now established the required property of $r_{3}, r_{5}, \ldots$ to make (5) hold, we return to the case of $r_{1}$, i.e. $n=1$ in (4). Note that $r_{1}=T$ is linear. By calculation, $g_{\theta}^{\prime \prime}(\beta)=\pi^{-4}\left(2 \pi^{2}-3 \theta^{2}\right)$, and $g_{\theta}^{\prime \prime}(0)<0$. Hence for $0 \leqslant \theta<\sqrt{2 / 3} \pi=\theta_{0}, g_{\theta}^{\prime \prime}$ has a zero at $\alpha$, $0<\alpha<\beta$. Since $g_{\theta}^{\prime}(x)<0(0<x<\beta)$ and $g_{\theta}^{\prime}(0)=0, \alpha$ is unique by Laguerre's theorem applied to $g_{\theta}^{\prime}$. Since $g_{\theta}^{\prime \prime}(x)<0 \quad(0<x<\alpha)$, we have $T(0)>g_{\theta}(0)>0$, and similarly $T(\beta)<g_{\theta}(\beta)=0$. Since $T$ has negative slope, $T>0$ at all negative zeros of $g_{\theta}$, and $T<0$ at all positive zeros of $g_{\theta}$. Therefore $t T(t)=\operatorname{tr}_{1}(t)<0$ at all these zeros. This proves (5).

Let $n \in \mathbb{N}, 0 \leqslant \theta<\theta_{0}$ and $Q^{2}=(z+\alpha)^{2}+\theta^{2}$. We know that

$$
e(z)=f_{\theta}(z+\alpha)=\cos Q
$$

satisfies $|\phi(e)|=\sup \left\{|\phi(f)|: f \in X_{1}\right\}$. Hence by Lemma $4,|\phi(e)|=\left\|h^{2 n+1}-i^{2 n+1} \tau\right\|$. Define $k(z)=e^{-i A}(\cos Q+i(A+\alpha z / A) \sin Q / Q)$. As in the even case, $k \in X$. For real $x$, $Q^{2}=x^{2}+2 \alpha x+A^{2}>(A+\alpha x / A)^{2}$, which gives $|k| \leqslant 1$ on $\mathbb{R}$ and so $k \in X_{1}$. Since $k(0)=1, \quad \xi=i^{2 n+1} k^{(2 n+1)}(0) \in V\left(h^{2 n+1}\right)$. Since $\sin Q=0$ at each $\alpha_{k} \neq-\alpha$, (4) gives $\phi((A+\alpha z / A) \sin Q / Q)=0$. Hence $\left|\zeta-\tau^{\prime}\right|=|\phi(k)|=|\phi(e)|=\left\|h^{2 n+1}-\tau^{\prime}\right\|$, where we put $\tau^{\prime}=i^{2 n+1} \tau$. Thus $\zeta \in \partial V\left(h^{2 n+1}\right)$, and $V\left(h^{2 n+1}\right) \subseteq$ the circle with centre $\tau^{\prime}$ and through $\zeta$.

We prove that $|\tau| \rightarrow \infty$ as $\theta \rightarrow \theta_{0}$ and so $A \rightarrow \pi$. We have $e^{\prime}(0)=-\alpha \sin A / A \rightarrow 0$ and $-e^{(2 n+2)}(0)=D_{x}^{2 n+1}[(x+\alpha) \sin Q / Q](0)=\alpha D_{x}^{2 n+1} \sin Q / Q(0)=\alpha g_{\theta}^{(2 n+1)}(\alpha)=\alpha(-1)^{n} G^{r}(\alpha)$ $\neq 0$, by (9), and since $D_{x}^{2 n} \sin Q / Q(0)=0$. This remains non-zero at $\theta_{0}$, and so 
$|\tau| \rightarrow \infty$. As $A \rightarrow \pi, \zeta \rightarrow \zeta_{0}$ where

$$
\pm \operatorname{Re}\left(\zeta_{0}\right)=D_{x}^{2 n+1}[(\pi+\alpha x / \pi) \sin Q / Q](0)=\pi D_{x}^{2 n+1} \sin Q / Q(0) \neq 0 .
$$

Since $\zeta \in V\left(h^{2 n+1}\right)$, so does $\zeta_{0}$. The function $\overline{k(-\bar{z})}$ gives $-\bar{\zeta}$ and $-\bar{\zeta}_{0}$ in $V$. The above circle centred at $\tau^{\prime}$ has $\left|\tau^{\prime}\right| . \rightarrow \infty$, and since $\tau^{\prime} \in i \mathbb{R}$, we get $\left[\zeta_{0},-\bar{\zeta}_{0}\right] \subseteq \partial V$, as before. Also, using the functions $\overline{k(\bar{z})}$, we get $\left[\bar{\zeta}_{0}, \zeta_{0}\right] \subseteq \partial V$. Note that $\zeta_{0} \neq-\bar{\zeta}_{0}$.

When $\theta=0, k(z)=e^{i z}$ and $\zeta=-1$. As $\theta$ varies from 0 to $\theta_{0}, \tau$ traces a continuous curve from -1 to $\xi_{0}$ in $\partial V$, since $A$ and $\alpha$ are continuous in $\theta$. The reflections of this arc in the axes and the origin are also in $\partial V$. With the two line segments they give a closed curve, which must be all of $\partial V$.

\section{REFERENCES}

1. R. P. Boas, Entire functions (Academic Press, 1954).

2. B. Bollobás, The numerical range in Banach algebras and complex functions of exponential type, Bull. London Math. Soc. 3 (1971), 27-33.

3. F. F. Bonsall and J. Duncan, Numerical ranges of operators on normed spaces and elements of normed algebras. London Math. Soc. Lecture Notes 2 (Cambridge University Press, 1971).

4. F. F. Bonsall and J. Duncan, Numerical ranges II, London Math. Soc. Lecture Notes 10 (Cambridge University Press, 1973).

5. A. Browder, States, Numerical ranges, etc., Proc. Brown Informal analysis Seminar, 1969.

6. A. Browder, On Bernstein's inequality and the norm of Hermitian operators, Amer. Math. Monthly 78 (1971), 871-873.

7. M. J. Crabb, J. Duncan and C. M. McGregor, Some extremal problems in the theory of numerical ranges, Acta Math. 128 (1972), 123-142.

8. P. R. Halmos, A Hilbert Space Problem book, (Van Nostrand, 1967).

9. G. H. Hardy, A Course of Pure Mathematics, (Cambridge, ed. 5, 1928).

10. Y. L. Luke, Integrals of Bessel functions, (McGraw-Hill, 1962). 